\title{
Integral inequality for time-varying delay systems
}

\author{
Alexandre Seuret ${ }^{1,2}$ and Frédéric Gouaisbaut ${ }^{1,3}$
}

\begin{abstract}
This paper considers the stability of time-varying delay systems. We develop some new integral inequalities which are proved to encompasses the celebrated Jensen's inequality. These technical tools allow to construct simple LyapunovKrasovskii functionals very efficient in practice. Notice that our procedure is coupled with the use of the reciprocal convexity result in order to reduce the conservatism induced by the LMIs optimisation setup. The effectiveness of the proposed results is illustrated by some classical examples from the literature.
\end{abstract}

\section{INTRODUCTION A REVOIR}

The delay phenomenon arises in many practical situations like in biology, economy or mechanical engineering (see for instance [8], [22]) and references therein) and therefore, since several years, it has motivated a huge number of results devoted to the construction of stability criteria for linear time delay systems. In the case of a constant delay and nominal system, some theoretical tools like direct eigenvalues analysis [22] are now well established and allow to derive efficient criteria with a relatively low numerical complexity. For the case of time-varying delay, two different methodologies have been employed. In the first framework, we aim at transforming the original system into a closed loop between a nominal system and a system depending on the delay. This last element is embedded into an uncertainty and the use of classical tools like Small Gain Theorem [7], [15], IQCs [12] or Quadratic Separation [3] allows to conclude on the stability. Another technique is the extension of Lyapunov theory to the case of delay systems. In a certain sense, all the results follow the same methodology [8]. Firstly, the initial retarded system is transformed into a more suitable equation. This transformation may not be equivalent with regards to the stability : the original system may be stable but not the transformed system [8]. Then, the structure of a LyapunovKrasovskii functional is proposed, of which aim is to prove the stability of the transformed system and the original one as well. This choice of this structure a priori often induces an important conservatism and several attempts has been proposed to reduce it by choosing extended state based Lyapunov-Krasovskii functional ([1], [13]), or discretized Lyapunov functional ([8]). The third step which brings an important source of conservatism comes from the inequalities to be used to derive a tractable numerical optimization problem (see [10], [11], [14], [18], [21], [23]. Generally, all the papers proceed in two stages: Firstly, all the cross terms of the derivative of $V$ are bounded using a Jensen's

\footnotetext{
1 CNRS, LAAS, 7 avenue du Colonel Roche, 31077 Toulouse, France aseuret, fgouaisbelaas.fr

${ }^{2}$ Univ de Toulouse, LAAS, F-31400 Toulouse, France.

3 Univ de Toulouse, UPS, LAAS, F-31400, Toulouse, France.
}

like inequality. Then, in order to get an LMI with respect to the delay $h(t)$ and the delay derivative $\dot{h}(t)$, the result is transformed into a convex optimization problem often with the help of slack variables [18]. In this paper, unlike many papers in the literature, we focus on the two last stage of the procedure, the development of less restrictive inequalities and the choice of slack variables. First of all, following the work of [14] or [4], we propose to construct more accurate inequalities than Jensen's or Wirtinger's ones. The resulting inequalities depend not only on the state $x(t)$ and the delayed state but also on the integral of the state over a delay interval. This new signal is then directly integrated into a suitable classical Lyapunov function, highlighting so the features of this new inequality. Then, we propose to extend the work of [18] in order to get tractable optimization scheme but with a fewer numbers of slack variables to be optimized.

The paper is organized as follows. Section 2 introduces a new integral inequality which can include the Jensen's one as a special case. We also propose a new reciprocally convex combination inequality in order to get some tractable convex optimisation problem to deal with. Section 3 is devoted to the use of classical Lyapunov-Krasovskii functional combined with inequalities describes in Section 2. Section 4 illustrates our results with some examples extracted from the literature.

Notations: Throughout the paper $\mathbb{R}^{n}$ denotes the $n$ dimensional Euclidean space with vector norm $|\cdot|, \mathbb{R}^{n \times m}$ is the set of all $n \times m$ real matrices. For any symmetric matrix $P \in \mathbb{R}^{n \times n}$, the notation $P \succ 0$ (or $P \prec 0$ ) means that $P$ is positive (or negative) definite. The set $\mathbb{S}_{+}^{n}$ refers to the set of symmetric positive definite matrices. The symmetric matrix $\left[\begin{array}{cc}A & B \\ * & C\end{array}\right]$ stands for $\left[\begin{array}{cc}A & B \\ B^{T} & C\end{array}\right]$. For any matrices $A, B$ in $\mathbb{R}^{n \times n}$, the notation $\operatorname{diag}(A, B)$ denotes the bloc diagonal matrix $\left[\begin{array}{ll}A & 0 \\ * & B\end{array}\right]$. Finally, a set of scalar parameter $\alpha_{1}, \ldots, \alpha_{N}, N$ belongs to the set $\mathcal{D}$, if each parameters is positive and $\sum_{i=1}^{N}=1$.

\section{SOME RELEVANT INEQUALITIES}

In this section, we aim at presenting several integral inequalities which will be employed in order to derive stability conditions for linear systems with time-varying delays. More specifically, we formulate a new inequality which is proved to be less conservative compared to Jensen's inequality. Furthermore, based on a reciprocally convex combination lemma provided in [18], we propose some new relaxations for this lemma which allows to reduce the number of variables involved in the optimization setup. 


\section{A. Improved integral inequality}

In the following subsection, we develop a new integral inequality which introduces less conservatism than the wellknown Jensen's inequality.

Lemma 2.1: For a given symmetric positive definite matrix $R \in \mathbb{S}_{n}^{+}$, any differentiable function $\omega$ in $[a, b] \rightarrow \mathbb{R}^{n}$, then the following inequality holds:

$$
\begin{aligned}
\int_{a}^{b} \dot{\omega}(u) R \dot{\omega}(u) \mathrm{d} u \geq & \frac{1}{b-a}(\omega(b)-\omega(a))^{T} R(\omega(b)-\omega(a)) \\
& +\frac{12}{b-a} \Omega^{T} R \Omega .
\end{aligned}
$$

where $\Omega=\frac{\omega(b)+\omega(a)}{2}-\frac{1}{b-a} \int_{a}^{b} \omega(u) \mathrm{d} u$.

Proof. For any sufficiently differentiable function $\omega$ in $[a, b] \rightarrow \mathbb{R}^{n}$, consider a signal $z$ given, for all $u \in[a, b]$ by

$$
z(u)=\dot{\omega}(u)-\frac{1}{b-a}(\omega(b)-\omega(a))+6 \frac{(b+a-2 u)}{(b-a)^{2}} \Omega,
$$

where $\Omega$ has been defined in the statements of the lemma The computation of $\int_{a}^{b} z^{T}(u) R z(u) \mathrm{d} u$ leads to:

$$
\begin{aligned}
\int_{a}^{b} z^{T}( & R) R z(u) \mathrm{d} u=\int_{a}^{b} \dot{\omega}^{T}(u) R \dot{\omega}(u) \mathrm{d} u \\
& +\frac{\int_{a}^{b} 1 \mathrm{~d} u}{(b-a)^{2}}(\omega(b)-\omega(a))^{T} R(\omega(b)-\omega(a)) \\
& +36 \frac{\int_{a}^{b}(b+a-2 u)^{2} \mathrm{~d} u}{(b-a)^{4}} \Omega^{T} R \Omega \\
& -\frac{2}{b-a} \int_{a}^{b} \dot{\omega}^{T}(u) \mathrm{d} u R(\omega(b)-\omega(a)) \\
& +\frac{12}{(b-a)^{2}} \int_{a}^{b}(b+a-2 u) \dot{\omega}^{T}(u) \mathrm{d} u R \Omega \\
& -\frac{12}{(b-a)^{3}} \int_{a}^{b}(b+a-2 u) \mathrm{d} u(\omega(b)-\omega(a))^{T} R \Omega .
\end{aligned}
$$

Simple calculus ensure that $\int_{a}^{b}(b+a-2 u)^{2} \mathrm{~d} u=\frac{1}{3}$ and $\int_{a}^{b}(b+a-2 u) \mathrm{d} u=0$. Then an integration by parts ensures that

$$
\int_{a}^{b}(b+a-2 u) \dot{\omega}^{T}(u) \mathrm{d} u=-2(b-a) \Omega .
$$

It thus follows that

$$
\begin{aligned}
\int_{a}^{b} z^{T}(u) & R z(u) \mathrm{d} u=\int_{a}^{b} \dot{\omega}^{T}(u) R \dot{\omega}(u) \mathrm{d} u \\
& +\frac{1}{(b-a)}(\omega(b)-\omega(a))^{T} R(\omega(b)-\omega(a)) \\
& -\frac{2}{(b-a)}(\omega(b)-\omega(a))^{T} R(\omega(b)-\omega(a)) \\
& +\frac{12}{(b-a)} \Omega^{T} R \Omega-\frac{24}{(b-a)} \Omega^{T} R \Omega .
\end{aligned}
$$

Since the matrix $R$ is positive definite, the left-hand side of the previous equation is positive definite. This allows us to conclude the proof of Lemma 2.1.

In order to compare with the celebrated Jensen's Lemma, let us recall this lemma, often at the root of several important results on the stability of time delay systems:

Lemma 2.2: (Jensen's Lemma) For given symmetric positive definite matrices $R>0$ and for any differentiable signal $\omega$ in $[a, b] \rightarrow \mathbb{R}^{n}$, the following inequality holds:

$$
\int_{a}^{b} \dot{\omega}(u) R \dot{\omega}(u) \mathrm{d} u \geq \frac{1}{b-a}(\omega(b)-\omega(a))^{T} R(\omega(b)-\omega(a)) .
$$

Proof. A proof can be found in [8],[16]. However the previous lemma allows to give an alternative proof by considering $\Omega=0$ in the definition of the function $z$.

Remark 1: The difference between the two inequalities are the following: in Lemma 2.1, the first term refers exactly to the right hand side of the Jensen's inequality provided in Lemma 2.2. The second term of (1) is positive definite. Thus it is clear that this new inequality encompasses the Jensen's inequality. It is also worth noting that this improvement is allowed by using an extra signal $\int_{a}^{b} \omega(u) \mathrm{d} u$ and not only the signals $\omega(b)$ and $\omega(a)$. Therefore, it suggests that in order to be useful, this inequality should be combined with a Lyapunov functional where the signal $\int_{a}^{b} \omega(u) \mathrm{d} u$ appears explicitly.

Remark 2: A similar integral inequality based on Wirtinger's inequality has been recently proposed in [19], [20]. The difference with respect to this inequality relies in the coefficient 12 which replaces the previously obtained coefficient $\pi^{2}$ (which is lower than 12). This new approach results in a less conservative result.

\section{B. Improved reciprocally convex combination inequality}

The following is largely inspired from the reciprocally convex combination lemma provided in [18]. Recall firstly an useful lemma provided in this article:

Lemma 2.3: [18] Let $f_{1}, f_{2}, \ldots, f_{N}: \mathbb{R}^{m} \rightarrow \mathbb{R}$ have positive values in an open subset $\mathcal{D}$ of $\mathbb{R}^{m}$. The reciprocally convex combination of $f_{i}$ over $\mathcal{D}$ satisfies

$$
\min _{\left.\left\{\alpha_{i}\right\}_{i} \in \mathcal{D}\right\}} \sum_{i} \frac{1}{\alpha_{i}} f_{i}(t)=\sum_{i} f_{i}(t)+\max _{g_{i j}(t)} \sum_{i} \sum_{j \neq i} g_{i j}(t),
$$

subject to

$$
\left\{g_{i j}: \mathbb{R}^{m} \rightarrow \mathbb{R}, g_{i j}(t) \triangleq g_{j i}(t),\left[\begin{array}{cc}
f_{i}(t) & g_{i j}(t) \\
g_{i j}(t) & f_{j}(t)
\end{array}\right] \succeq 0\right\}
$$

An application of this lemma which was already proposed in the same article is stated as follows.

Lemma 2.4: Let $n, m$ be two integers, $R$ a symmetric matrices in $\mathbb{S}^{n}$ and two matrices $W_{1}$ and $W_{2}$ in $\mathbb{R}^{n \times n m}$. Consider a scalar $\alpha$ in the interval $(0,1)$. For all vector $\xi$ in $\mathbb{R}^{n m}$, define the function $\Theta(\alpha, R)$ as:

$$
\Theta(\alpha, R)=\frac{1}{\alpha} \xi^{T} W_{1}^{T} R W_{1} \xi+\frac{1}{1-\alpha} \xi^{T} W_{2}^{T} R W_{2} \xi .
$$

Then the improved reciprocally convex combination guarantees that if there exists a matrix $X$ in $\mathbb{R}^{n \times n}$ such that

$$
\left[\begin{array}{cc}
R & X \\
* & R
\end{array}\right] \succ 0
$$

then the following inequality holds

$$
\min _{\alpha \in(0,1)} \Theta(\alpha, R) \geq\left[\begin{array}{l}
W_{1} \xi \\
W_{2} \xi
\end{array}\right]^{T}\left[\begin{array}{cc}
R & X \\
* & R
\end{array}\right]\left[\begin{array}{l}
W_{1} \xi \\
W_{2} \xi
\end{array}\right] .
$$

Proof. The proof is omitted but can be found in [18].

In the following, an alternative lemma is proposed in order to reduce the number of the slack variables by eliminating the matrix $X$.

Lemma 2.5: Let $n, m$ be two integers, $R$ a symmetric matrix in $\mathbb{S}^{n}$ and two matrices $W_{1}$ and $W_{2}$ in $\mathbb{R}^{n \times n m}$. Consider a scalar $\alpha$ in the interval $(0,1)$. Then the improved reciprocally convex combination guarantees that

$$
\min _{\alpha \in(0,1)} \Theta(\alpha, R) \geq \xi^{T}\left[W_{1}-W_{2}\right]^{T} R\left[W_{1}-W_{2}\right] \xi .
$$


Proof. As mentioned in the introduction of the section, the proof is is based on the main result of [18] but adapted to our case in order to reduce the number of slack variables. Following [18], for all $\xi$ in $\mathbb{R}^{n m}$, the function $\Theta(\alpha, R)$ can be rewritten as

$$
\begin{aligned}
\Theta(\alpha, R)= & \frac{\alpha+(1-\alpha)}{\alpha} \xi^{T} W_{1}^{T} R W_{1} \xi \\
& +\frac{\alpha+(1-\alpha)}{1-\alpha} \xi^{T} W_{2}^{T} R W_{2} \xi \\
= & \xi^{T} W_{1}^{T} R W_{1} \xi+\xi^{T} W_{2}^{T} R W_{2} \xi \\
& +\frac{1-\alpha}{\alpha} \xi^{T} W_{1}^{T} R W_{1} \xi+\frac{\alpha}{1-\alpha} \xi^{T} W_{2}^{T} R W_{2} \xi
\end{aligned}
$$

By noting that $\frac{1-\alpha}{\alpha}$ and its inverse are positive, one can rewrite the last term of the previous equation as follows

$$
\begin{aligned}
\Theta(\alpha, R)= & \xi^{T} W_{1}^{T} R W_{1} \xi+\xi^{T} W_{2}^{T} R W_{2} \xi \\
& -2 \xi^{T} W_{1}^{T} R W_{2} \xi \\
& +\left(\sqrt{\frac{1-\alpha}{\alpha}} W_{1} \xi+\sqrt{\frac{\alpha}{1-\alpha}} W_{2} \xi\right)^{T} R \\
& \times\left(\sqrt{\frac{1-\alpha}{\alpha}} W_{1} \xi+\sqrt{\frac{\alpha}{1-\alpha}} W_{2} \xi\right) .
\end{aligned}
$$

Since the matrix $R$ is positive definite, the last term of the previous expression is positive definite. Then it yields

$$
\begin{aligned}
\Theta(\alpha, R) \geq & \xi^{T} W_{1}^{T} R W_{1} \xi+\xi^{T} W_{2}^{T} R W_{2} \xi \\
& -2 \xi^{T} W_{1}^{T} R W_{2} \xi,
\end{aligned}
$$

which concludes the proof

Remark 3: Obviously, our proposed new inequality is more conservative that the inequality from Lemma 2.4. Nevertheless, this advantage should be balanced with the numerical burden generated by the slack variable $X$ as we will see with the numerical simulations.

In the sequel, these two lemmas will be employed in order to derive stability conditions for linear systems with timevarying delay. In particular a comparison on their use and on their induced conservatism will be discussed.

\section{STABILITY ANALYSIS OF TIME-VARYING DELAY SYSTEMS}

We present in this sub-section a first stability result for time delay systems, which is based on the use of Jensen's inequality. Consider a linear time-delay system of the form:

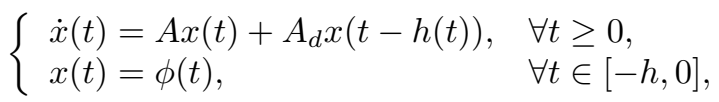

where $x(t) \in \mathbb{R}^{n}$ is the state vector, $\phi$ is the initial condition and $A, A_{d}, A_{D} \in \mathbb{R}^{n \times n}$ are constant matrices. The delay is assumed to be time-varying. The classical constraints on the delay function are

$$
\begin{aligned}
& h(t) \in\left[0, h_{M}\right] \\
& \dot{h}(t) \in\left(-\infty, d_{M}\right], \quad \forall t \geq 0,
\end{aligned}
$$

for some scalars $0 \leq h_{M}$ and $d_{M} \leq 1$.

Based on the previous inequality and classical results on Lyapunov-Krasovskii functionals, three stability theorems are provided. The two first results are based on the use of the Jensen's inequality and Lemma 2.4 and 2.5. The last one explicitly use the new inequality together with the reciprocally convex combination lemma provided in [18].

\section{A. Stability analysis based on the Jensen's inequality}

The stability analysis of the time-delay system (7) are established is the following theorems.

Theorem 1: Assume that there exist two scalars $h_{M}>0$, $d_{M}>0$ and some matrices $P, S, R, Q$ in $\mathbb{S}_{+}^{n}$ and $X$ in $\mathbb{R}^{n \times n}$ such that the following LMIs are satisfied

$$
\begin{aligned}
& \Theta_{0}=\left[\begin{array}{cc}
R & X \\
* & R
\end{array}\right] \quad \succ 0 \\
& \Psi_{1}\left(h_{M}, d_{M}\right)=\Psi_{0}\left(d_{M}\right)-\frac{1}{h_{M}} \Pi_{0}^{T} \Theta_{0} \Pi_{0} \prec \quad \prec,
\end{aligned}
$$

where

$$
\begin{aligned}
\Psi_{0}\left(d_{M}\right)= & F_{1}^{T} P F_{0}+F_{0}^{T} P F_{1}+\hat{S} \\
& +\hat{Q}\left(d_{M}\right)+h_{M} F_{0}^{T} R F_{0}
\end{aligned}
$$

with

$$
\begin{array}{ll}
\hat{Q}\left(d_{M}\right) & =\operatorname{diag}\left(Q,-\left(1-d_{M}\right) Q, 0\right), \\
\hat{S} & =\operatorname{diag}(S, 0,-S), \\
\Pi_{0} & =\left[\begin{array}{l}
F_{2} \\
F_{3}
\end{array}\right]
\end{array}
$$

and

$$
\begin{aligned}
& F_{0}=\left[\begin{array}{lll}
A & A_{d} & 0
\end{array}\right], \quad F_{1}=\left[\begin{array}{lll}
I & 0 & 0
\end{array}\right], \\
& F_{2}=\left[\begin{array}{lll}
I & -I & 0
\end{array}\right], \quad F_{3}=\left[\begin{array}{lll}
0 & -I & I
\end{array}\right] .
\end{aligned}
$$

Then the system (7) is asymptotically stable for the timevarying delay $h$ satisfying (8).

Notice that the last Theorem 1 does not depend on the lower bound of $\dot{h}(t)$.

Remark 4: It has to be noticed that Theorem 1 is similar to the one provided in [18] without lower bound of the delay and with $d_{M}=1$.

The second theorem is formulated as follows:

Theorem 2: Assume that there exist two scalars $h_{M}>0$, $d_{M}>0$ and some matrices $P, S, R, Q$ in $\mathbb{S}_{+}^{n}$, such that the following LMI is satisfied

$$
\Psi_{2}\left(h_{M}, d_{M}\right)=\Psi_{0}\left(d_{M}\right)-\frac{1}{h_{M}} \Pi_{1}^{T} R \Pi_{1} \prec 0,
$$

where $\Psi_{0}\left(d_{M}\right)$ is defined in (9) and where $\Pi_{1}=F_{2}-F_{3}$. Then the system (7) is asymptotically stable for the timevarying delay $h$ satisfying (8).

Proof. Consider the Lyapunov functional given by

$$
\begin{aligned}
V\left(h, x_{t}, \dot{x}_{t}\right)= & x^{T}(t) P x(t)+\int_{t-h_{M}}^{t} x^{T}(s) S x(s) \mathrm{d} s \\
& +\int_{t-h(t)}^{t} x^{T}(s) Q x(s) \mathrm{d} s \\
& +\int_{t-h_{M}}^{t}\left(h_{M}-t+s\right) \dot{x}^{T}(s) R \dot{x}(s) \mathrm{d} s .
\end{aligned}
$$

This functional is positive definite since the matrices $P$, $S, Q$ and $R$ are symmetric positive definite. It has to be noticed that the functional is very simple compared to some functionals that can be found in the literature. Differentiating the functional (12) along the trajectories of (7) leads to:

$$
\dot{V}\left(h, x_{t}, \dot{x}_{t}\right)=\xi^{T}(t) \Phi_{0}(\dot{h}) \xi(t)-\int_{t-h_{M}}^{t} \dot{x}^{T}(s) R \dot{x}(s) \mathrm{d} s,
$$


where $\xi(t)=\left[\begin{array}{lll}x^{T}(t) & x^{T}(t-h(t)) & x^{T}\left(t-h_{M}\right)\end{array}\right]^{T}$. This last equation has been obtained by noting that

$$
x(t)=F_{1} \xi(t), \quad \dot{x}(t)=F_{0} \xi(t) .
$$

Splitting the integral into two integrals defined over the intervals $[t-h(t), t]$ and $\left[t-h_{M}, t-h(t)\right]$ and applying the Jensen's inequality 2.2 , we get that

$$
\begin{aligned}
& \int_{t-h_{M}}^{t} \dot{x}^{T}(s) R \dot{x}(s) \mathrm{d} s \geq \\
& \quad \frac{1}{h(t)}[x(t)-x(t-h(t))]^{T} R[x(t)-x(t-h(t))] \\
& \quad \frac{1}{h_{M}-h(t)}\left[x(t-h(t))-x\left(t-h_{M}\right)\right]^{T} R \\
& \left.\quad \times\left[x(t-h(t))-x\left(t-h_{M}\right)\right)\right] .
\end{aligned}
$$

Rewriting the previous expression using the vector $\xi_{0}(t)$ and the matrices $F_{2}$ and $F_{3}$ yields to

$$
\int_{t-h(t)}^{t} \dot{x}^{T}(s) R \dot{x}(s) \mathrm{d} s \geq \xi^{T}(t) \frac{F_{2}^{T} R F_{2}}{h(t)} \xi(t) .
$$

Applying the same method to the second integral, it yields to

$$
\dot{V}\left(x_{t}, \dot{x}_{t}\right) \leq \xi^{T}(t)\left[\Phi_{0}(\dot{h})-\frac{F_{2}^{T} R F_{2}}{h(t)}-\frac{F_{3}^{T} R F_{3}}{h_{M}-h(t)}\right] \xi(t) .
$$

The right hand side of the previous inequality is of the appropriate form to apply Lemma 2.4 and Lemma 2.5 which leads to the stability conditions given in Theorems 1 and 2, respectively.

\section{B. Stability analysis based on the new integral inequality}

In this section, the assumptions on the delay function $h$ are modified. In the following, the also classical constraints on the delay function becomes

$$
\begin{aligned}
& h(t) \in\left[h_{m}, h_{M}\right] \\
& \dot{h}(t) \in\left[d_{m}, d_{M}\right]
\end{aligned}, \quad \forall t \geq 0,
$$

for some scalars $0 \leq h_{m} \leq h_{M}$ and $d_{m} \leq d_{M} \leq 1$.

Based on the previous inequality and classical results on Lyapunov-Krasovskii functionals, the stability theorem is provided.

Theorem 3: Assume that there exist matrices $P$ in $\mathbb{S}_{+}^{3 n}$, $S, Q, R$ in $\mathbb{S}_{+}^{n}$, and a matrix $X$ in $\mathbb{R}^{2 n \times 2 n}$. such that the following LMIs are satisfied for $h$ in $\left\{h_{m}, h_{M}\right\}$ and for $\dot{h}$ in $\left\{d_{m}, d_{M}\right\}$

$$
\begin{aligned}
& \Theta_{2} \quad=\left[\begin{array}{cc}
\tilde{R} & X \\
* & \tilde{R}
\end{array}\right] \quad \succ 0, \\
& \Phi(h, \dot{h})=\Phi_{0}(h, \dot{h})-\frac{1}{h_{M}} \Gamma^{T} \Theta_{2} \Gamma \prec 0,
\end{aligned}
$$

where

$$
\begin{aligned}
\Psi_{0}(h, \dot{h})= & G_{1}^{T}(h) P G_{0}(\dot{h})+G_{0}^{T}(\dot{h}) P G_{1}(h) \\
& +\hat{S}+\hat{Q}(\dot{h})+h_{M} G_{0}^{T}(\dot{h}) \hat{R} G_{0}(\dot{h}), \\
\hat{Q}(\dot{h})= & \operatorname{diag}\left(Q,-(1-\dot{h}) Q, 0_{3 n}\right), \\
\hat{S}= & \operatorname{diag}\left(S, 0,-S, 0_{2 n}\right), \\
\hat{R}= & \operatorname{diag}\left(R, 0_{3 n}\right), \\
\tilde{R}= & \operatorname{diag}(R, R),
\end{aligned}
$$

and

$$
\begin{aligned}
& G_{0}(\dot{h})=\left[\begin{array}{ccccc}
A & A_{d} & 0 & 0 & 0 \\
I & -(1-\dot{h}) I & 0 & 0 & 0 \\
0 & (1-\dot{h}) I & -I & 0 & 0
\end{array}\right], \\
& G_{1}(h)=\left[\begin{array}{ccccc}
I & 0 & 0 & 0 & 0 \\
0 & 0 & 0 & h I & 0 \\
0 & 0 & 0 & 0 & \left(h_{M}-h\right) I
\end{array}\right] \\
& \Gamma=\left[\begin{array}{cccc}
G_{2}^{T} & G_{3}^{T} & G_{4}^{T} & G_{5}^{T}
\end{array}\right]^{T}, \\
& G_{2}=\left[\begin{array}{lllll}
I & -I & 0 & 0 & 0
\end{array}\right] \text {, } \\
& G_{3}=\sqrt{3}\left[\begin{array}{lllll}
I & I & 0 & -2 I & 0
\end{array}\right], \\
& G_{4}=\left[\begin{array}{ccccc}
0 & -I & I & 0 & 0
\end{array}\right] \text {, } \\
& G_{5}=\sqrt{3}\left[\begin{array}{lllll}
0 & I & I & 0 & -2 I
\end{array}\right] \text {. }
\end{aligned}
$$

Then the system (7) is asymptotically stable for the timevarying delay $h$ satisfying (14).

Proof. Consider the Lyapunov-Krasovskii functional given by

$$
\begin{aligned}
V\left(h, x_{t}, \dot{x}_{t}\right)= & \zeta_{0}^{T}(t) P \zeta_{0}(t)+\int_{t-h(t)}^{t} x^{T}(s) Q x(s) \mathrm{d} s \\
& \int_{t-h_{M}}^{t} x^{T}(s) S x(s) \mathrm{d} s \\
& +\int_{t-h_{M}}^{t}\left(h_{M}-t+s\right) \dot{x}^{T}(s) R \dot{x}(s) \mathrm{d} s
\end{aligned}
$$

where

$$
\zeta_{0}(t)=\left[\begin{array}{c}
x(t) \\
\int_{t-h(t)}^{t} x(s) \mathrm{d} s \\
\int_{t-h_{M}}^{t-h(t)} x(s) \mathrm{d} s
\end{array}\right] .
$$

This functional is positive definite since the matrices $P, S$ and $R$ are symmetric positive definite. This functional is simple compared to some functionals that can be found in the literature. The only novelty remains in the introduction of the signals $\int_{t-h(t)}^{t} x(s) \mathrm{d} s$ and $\int_{t-h_{M}}^{t-h(t)} x(s) \mathrm{d} s$ in the functional which recall the method originally proposed by [8]. Differentiating the functional (17) along the trajectories of (7) leads to:

$$
\dot{V}\left(h, x_{t}, \dot{x}_{t}\right)=\zeta^{T}(t) \Phi_{0}(h) \zeta(t)-\int_{t-h_{M}}^{t} \dot{x}^{T}(s) R \dot{x}(s) \mathrm{d} s
$$

where

$$
\zeta(t)=\left[\begin{array}{c}
x(t) \\
x(t-h(t)) \\
x\left(t-h_{M}\right) \\
\frac{1}{h(t)} \int_{t-h(t)}^{t} x(s) \mathrm{d} s \\
\frac{1}{h_{M}-h(t)} \int_{t-h_{M}}^{t-h(t)} x(s) \mathrm{d} s
\end{array}\right] .
$$

This equation has been obtained by noting that

$$
\zeta_{0}(t)=G_{1}(h) \zeta(t), \quad \dot{\zeta}(t)=G_{0}(\dot{h}) \zeta(t) .
$$

Following the same procedure as in Theorem 2, the use of Lemma 2.1 leads to

$$
\begin{aligned}
& \int_{t-h_{M}}^{t} \dot{x}^{T}(s) R \dot{x}(s) \mathrm{d} s \geq \\
& \quad \frac{1}{h(t)} \zeta^{T}(t)[x(t)-x(t-h(t))]^{T} R[x(t)-x(t-h(t))] \\
& \quad \frac{12}{h(t)}\left[\frac{x(t)+x(t-h(t))}{2}-\frac{1}{h(t)} \int_{t-h(t)}^{t} x(s) \mathrm{d} s\right]^{T} R \\
& \quad \times\left[\frac{x(t)+x(t-h(t))}{2}-\frac{1}{h(t)} \int_{t-h(t)}^{t} x(s) \mathrm{d} s\right] .
\end{aligned}
$$


Then applying again Lemma 2.1 to the second integral and rewriting the previous expression using the vector $\zeta(t)$ and the matrices $G_{i}$ for $i=2, \ldots 5$ yields to

$$
\begin{aligned}
\int_{t-h_{M}}^{t} \dot{x}^{T}(s) R \dot{x}(s) \mathrm{d} s \geq & \zeta^{T}(t) \frac{\left(G_{2}^{T} R G_{2}+G_{3}^{T} R G_{3}\right)}{h(t)} \zeta(t) \\
& +\zeta^{T}(t) \frac{\left(G_{4}^{T} R G_{4}+G_{5}^{T} R G_{5}\right)}{h_{m}-h(t)} \zeta(t) .
\end{aligned}
$$

Applying the same method to the second integral and replacing the integral in (18) by its bounds, it yields

$$
\begin{array}{r}
\dot{V}\left(x_{t}, \dot{x}_{t}\right) \leq \zeta^{T}(t)\left[\Phi_{0}(h, \dot{h})-\frac{\left(G_{2}^{T} R G_{2}+G_{3}^{T} R G_{3}\right)}{h(t)}\right. \\
\left.-\frac{\left(G_{4}^{T} R G_{4}+G_{5}^{T} R G_{5}\right)}{h_{m}-h(t)}\right] \zeta(t) .
\end{array}
$$

The right hand side of the previous inequality is exactly of the form described in Lemma 2.5. According to Lemma 2.4, the derivative of the Lyapunov Krasovskii functional is negative definite if

$$
\Phi(h, \dot{h})<0, \forall(h, \dot{h}(t)) \in\left[0, h_{M}\right] \times\left[d_{m}, d_{M}\right] .
$$

As the matrix $\Phi(h, \dot{h})$ is linear with respect to $h(t)$ and $\dot{h}(t)$, a sufficient condition is to test the condition on its vertices, which concludes the proof. the conditions of Theorem 3 are satisfied.

Remark 5: The Lyapunov-Krasovskii functionals employed to derive the stability condition of Theorem 3 is very simple compared to the one that can be found in the literature (see for instance [21], [23] among many others). However, the use of the inequality proposed in Lemma 2.1 leads to a considerable reduction of the conservatism. This will be shown in the example section.

Remark 6: Any results based on the new inequality proposed by Lemma 2.1 combined with the reciprocal convexity result of Lemma 2.5 will be provided in the present paper. Indeed, the combination of these two lemmas does not give efficient stability conditions compared to Theorem 3 .

\section{EXAMPLES}

The purpose of the following section is to show how the previous theorems can be relevant for the stability analysis of linear systems with time-varying delays. Especially, we will show that Theorems 1 and 2 deliver similar results and that Theorem 3 provides with less conservative results than existing ones.

\section{A. Example 1}

Let consider the following linear time-delay system (7) with:

$$
A=\left[\begin{array}{cc}
-2 & 0 \\
0 & -0.9
\end{array}\right], \quad A_{d}=\left[\begin{array}{cc}
-1 & 0 \\
-1 & -1
\end{array}\right]
$$

This system is a well-known delay dependent stable system, that is the delay free system is stable and the maximum allowable delay $h_{\max }=6.1721$ can be easily computed by delay sweeping techniques. To show the effectiveness of our approach, results are compared to the literature and are reported in Table I for the constant $\left(d_{m}=d_{M}=0\right)$ and time-varying delay case.

\begin{tabular}{|c|c|c|c|c|c|c|}
\hline$d_{M}$ & 0 & 0.1 & 0.2 & 0.5 & 0.8 & 1 \\
\hline \hline$[5],[24]$ & 4.472 & 3.604 & 3.033 & 2.008 & 1.364 & 0.999 \\
\hline$[6]$ & 1.632 & 1.632 & 1.632 & 1.632 & 1.632 & 1.632 \\
\hline$[9],[10]$ & 4.472 & 3.605 & 3.039 & 2.043 & 1.492 & 1.345 \\
\hline$[23]$ & 4.472 & 3.611 & 3.047 & 2.072 & 1.590 & 1.529 \\
\hline$[8](\mathrm{N}=1)$ & 6.059 & $-^{1}$ & $-{ }^{1}$ & $-{ }^{1}$ & $-{ }^{1}$ & $-^{1}$ \\
\hline$[1]$ & 5.120 & 4.081 & 3.448 & 2.528 & 2.152 & 1.991 \\
\hline$[17](\mathrm{N}=4)$ & $--^{2}$ & 4.35 & $-{ }^{2}$ & 1.62 & $-{ }^{2}$ & $-{ }^{2}$ \\
\hline$[20]$ & 5.901 & 4.525 & 3.626 & 2.095 & 1.524 & 1.258 \\
\hline$[12]$ & 6.117 & 4.714 & 3.807 & 2.280 & 1.608 & 1.360 \\
\hline$[2]$ & 6.117 & 4.794 & 3.995 & 2.682 & 1.957 & 1.602 \\
\hline \hline Th.1 & 4.472 & 3.658 & 3.163 & 2.337 & 1.934 & 1.868 \\
\hline Th.2 & 4.433 & 3.657 & 3.163 & 2.337 & 1.934 & 1.868 \\
\hline Th.3 & 6.059 & 4.703 & 3.834 & 2.420 & 2.137 & 2.128 \\
\hline \multicolumn{7}{|c|}{ TABLE I } \\
\hline
\end{tabular}

THE MAXIMAL ALLOWABLE DELAYS $h_{M}$ FOR SYSTEM DESCRIBED IN EXAMPLE (20).

The conditions of Theorems 1 and 2 delivers similar results when the upper bound of $\dot{h}(t)$ is greater than 0.2 . It is no more the case, when the delay function varies slowly : the conditions issued from Theorem 1 give an upper bound for $\dot{h}(t)$ slightly greater than those given using Theorem 2 .

Hence, on this example, it seems that the use of Lemma 2.5 is more interesting for fast varying delays, since it also reduces the number of variables and of LMIs to be solved in the stability conditions.

Consider now the stability conditions provided by Theorem 3. All papers except [12], [1], [2] provide more conservative results than the ones from Theorem 3 . However when the bound on the derivative of the delay is greater than 0.8 , the condition from Theorem 3 becomes more efficient. Notice that the results [12], [1], [2] are based on a robust analysis setup. In that case, the original system is modeled by an extended closed loop system and classical tools like Small Gain Theorem, IQCs are used to give LMIs conditions. It involves generally a bigger number of variables to be optimized compared to our simple LyapunovKrasovskii functional.

\section{B. Example 2}

Theorem 3 addresses also the stability of systems with interval delays, which may be unstable for small delays (or without delays) as it is illustrated with the second example.

$$
A=\left[\begin{array}{cc}
0 & 1 \\
-2 & 0.1
\end{array}\right], \quad A_{d}=\left[\begin{array}{ll}
0 & 0 \\
1 & 0
\end{array}\right] \text {. }
$$

As $\operatorname{Re}\left(\operatorname{eig}\left(A+A_{d}\right)\right)=0.05>0$, the delay free system is unstable and in this case, the results to assess stability of this system are much more scarce. For the constant delay case, a frequency approach shows that the solutions of this system are stable if the delay belongs to the interval ] $0.10017,1.7178[$. It is worth noting that classical LyapunovKrasovskii approaches based on Jensen's inequality cannot 


\begin{tabular}{|c|c|c|}
\hline Theorems & $h_{\min }$ & $h_{\max }$ \\
\hline \hline$[10]$ & $\emptyset$ & $\emptyset$ \\
{$[3]$} & 0.102 & 1.424 \\
{$[8](\mathrm{N}=1)$} & 0.1006 & 1.4272 \\
\hline Th.3 & 0.1006 & 1.55 \\
\hline
\end{tabular}

TABLE II

RESULTS FOR EXAMPLE (21) FOR CONSTANT AND KNOWN DELAY

$$
h \in\left[h_{\min }, h_{\max }\right] .
$$

\begin{tabular}{|c|c|c|c|c|c|c|}
\hline$d_{M}$ & 0.0 & 0.1 & 0.2 & 0.5 & 0.8 & 1.0 \\
\hline$\delta$ & 0.552 & 0.528 & 0.493 & 0.278 & 0.189 & 0.161 \\
\hline \multicolumn{7}{c|}{ TABLE III }
\end{tabular}

RELATION BETWEEN $\delta$ AND $d_{M}$ PROVIDE BY THEOREM 3 FOR EXAMPLE (21) WITH A TIME-VARYING DELAY $h \in[0.9-\delta, 0.9+\delta]$

$$
\text { AND } d_{m}=-d_{M} \text {. }
$$

assess stability of such systems. This is the case when applying the LMI conditions from theorems 2 and 1. However the stability condition of Theorem 3 have solutions thanks to the use of the new integral inequality provided in Lemma 2.1.

1) Constant and known delay: The results obtained by solving the conditions from Theorem 3 are resumed in the following tables. Tables II shows the results when the delay function is constant and known, i.e. $h_{m}=h_{M}$ and $d_{m}=$ $d_{M}=0$. Note that only few methods from the literature are able to provide relevant results for this systems. The table shows that Theorem 3 provides stability conditions which are more tight than the one given in the literature.

2) Time-varying delay: Table III shows the results provided by Theorem 3 in the case of time-varying delay. In order to show the results in an efficient way, the delay function is rewritten as

$$
h(t)=h_{a v}+\Delta h(t)
$$

where

$$
h_{a v}=0.9 \simeq(0.10017+1.7178) / 2,
$$

and

$$
\|\Delta h(t)\| \leq \delta
$$

Using such delay representation, the parameter $2 \delta$ represents the length of the interval to which $h(t)$ belongs. It is worth noting that the Sum of Square method provided in [17] is also able to characterize stability of such system. However we were not able to show their results in the present paper.

\section{Conclusions}

This article presents a new integral inequality which has been proved to be suitable for the stability analysis of timedelay systems. Combining it with the reciprocal convexity lemma leads to less conservative stability conditions than the usual ones which consider the Lyapunov-Krasovskii approach. It is also shown that for fast-varying delays, the proposed method is less conservative than the ones which are based on IQC's. Furthers research aims at reducing the conservatism with respect to the IQC approach for slowvarying delays.

\section{REFERENCES}

[1] Y. Ariba and F. Gouaisbaut. An augmented model for robust stability analysis of time-varying delay systems. Int. J. Control, 82:1616-1626, September 2009.

[2] Y. Ariba and F. Gouaisbaut. Input-output framework for robust stability of time-varying delay systems. In the 48th IEEE Conference on Decision and Control (CDC'09), Shanghai, China, December 2009.

[3] Y. Ariba, F. Gouaisbaut, and K.H. Johansson. Stability interval for time-varying delay systems. In Decision and Control (CDC), 2010 49th IEEE Conference on, pages 1017 -1022, dec. 2010.

[4] C. Briat. Convergence and equivalence results for the Jensen's inequality - application to time-delay and sampled-data systems. IEEE Transactions on Automatic Control, 56(7):1660-1665, 2011.

[5] E. Fridman and U Shaked. An improved stabilization method for linear time-delay systems. IEEE Trans. on Automat. Control, 47:1931-1937, November 2002.

[6] E. Fridman and U Shaked. Input-output approach to stability and $l_{2}$ gain analysis of systems with time-varying delays. Systems \& Control Letters, 55:1041-1053, September 2006.

[7] H. Fujioka. Stability analysis of systems with aperiodic sample-andhold devices. Automatica, 45(3):771-775, 2009

[8] K. Gu, V.-L. Kharitonov, and J. Chen. Stability of time-delay systems. Birkhauser, 2003.

[9] Y. He, Q. G. Wang, C. Lin, and M. Wu. Delay-range-dependent stability for systems with time-varying delay. Automatica, 43:371376, 2007.

[10] Y. He, Q. G. Wang, L. Xie, and C. Lin. Further improvement of free-weighting matrices technique for systems with time-varying delay. IEEE Trans. on Automat. Control, 52:293-299, February 2007.

[11] X. Jiang and Q.L. Han. Delay-dependent robust stability for uncertain linear systems with interval time-varying delay. Automatica, 42(6):1059-1065, 2006.

[12] C.Y. Kao and A. Rantzer. Stability analysis of systems with uncertain time-varying delays. Automatica, 43(6):959 - 970, 2007.

[13] J.H. Kim. Note on stability of linear systems with time-varying delay. Automatica, 47(9):2118-2121, 2011.

[14] K. Liu and E. Fridman. Wirtinger's inequality and lyapunov-based sampled-data stabilization. Automatica, 48(1):102108, 2012.

[15] L. Mirkin. Some remarks on the use of time-varying delay to model sample-and-hold circuits. IEEE Trans. on Automatic Control, 52(6):1009-1112, 2007.

[16] S.-I. Niculescu. Delay Effects on Stability. A Robust Control Approach. Springer-Verlag, 2001.

[17] A. Papachristodoulou, M. M. Peet, and S.-I. Niculescu. Stability analysis of linear systems with time-varying delays: Delay uncertainty and quenching. In Proc. of the $46^{\text {th }}$ IEEE Conference on Decision and Control, New Orleans, LA, USA, Dec. 12-14, 2007.

[18] P.G. Park, J. W. Ko, and C. Jeong. Reciprocally convex approach to stability of systems with time-varying delays. Automatica, 47:235238, 2011.

[19] A. Seuret and F. Gouaisbaut. On the use of the wirtinger's inequalities for time-delay systems. In Proc. of the $10^{\text {th }}$ IFAC Workshop on Time Delay Systems (IFAC TDS'12), Boston, MA, USA, 2012.

[20] A. Seuret and F. Gouaisbaut. Reducing the gap of the jensen's inequality by using the wirtinger's inequality. submitted to Automatica, 2012.

[21] H. Shao. New delay-dependent stability criteria for systems with interval delay. Automatica, 45(3):744 - 749, 2009.

[22] R. Sipahi, S. Niculescu, C.T. Abdallah, W. Michiels, and K. Gu. Stability and stabilization of systems with time delay. Control Systems, IEEE, 31(1):38 -65, feb. 2011.

[23] J. Sun, G.P. Liu, J. Chen, and D. Rees. Improved delay-rangedependent stability criteria for linear systems with time-varying delays. Automatica, 46(2):466 - 470, 2010.

[24] M. Wu, Y. He, J. H. She, and G. P. Liu. Delay-dependent criteria for robust stability of time-varying delay systems. Automatica, 40:14351439, 2004. 